Rev. Adm. Saúde (On-line), São Paulo, v. 19, n. 76: e185, jul. - set. 2019, Epub 09 set. 2019 http://dx.doi.org/10.23973/ras.76.185

ARTIGO ORIGINAL

\title{
Podem os princípios do ikigai contribuir para os programas de qualidade de vida no ambiente de trabalho?
}

Can ikigai principles contribute to quality of life programs in the workplace?

\author{
Milton M. Osaki ${ }^{1}$
}

1. Médico, especialista em administração em saúde. Presidente da Sociedade Paulista de Medicina Preventiva e Administração em Saúde (SOMPAS)

\section{RESUMO}

O ikigai representa a visão de vida, a sensibilidade e a maneira de agir dos japoneses, levando ao bem-estar e longevidade do indivíduo, através do incentivo a atividade física, social, alimentação frugal, manutenção do trabalho e dedicação às paixões, dando um sentido à vida.

A qualidade de vida no trabalho é desenvolvida nas organizações através dos programas de segurança e saúde ocupacional, e seus consequentes programas, como o programa de promoção de hábitos saudáveis (atividade física regular, alimentação saudável e lazer) e o programa de preparação para a aposentadoria.

Assim, nos programas de qualidade de vida, em especial no programa de preparação para aposentadoria, os trabalhadores têm a possibilidade de refletir sobre o momento presente, sem esquecer o fim do período produtivo e envelhecimento, a discussão do ideário do ikigai cabe como proposta e alternativa para conhecimento das ações necessárias para aumentar as possibilidades de maior bem-estar físico, emocional, mental e espiritual.

Para a incorporação do ideário do ikigai em cada um, talvez, não sejam necessárias grandes mudanças no estilo de vida. A necessidade seria entender a essência do conceito e incorporá-lo ao cotidiano. Seria descobrir a "paixão", descobrir aquilo que traz a felicidade. 


\section{ABSTRACT}

Ikigai represents the Japanese view of life, sensitivity, and way of acting, leading to the well-being and longevity of the individual by encouraging physical, social activity, frugal eating, job maintenance and dedication to the passions, giving a meaning to life.

Quality of life at work is developed in organizations through occupational health and safety programs, and their consequent programs such as the healthy habits promotion program (regular physical activity, healthy eating and leisure) and the retirement preparation program.

Thus, in the quality of life programs, especially the retirement preparation program, workers have the possibility to reflect on the present moment, without forgetting the end of the productive period and aging, the discussion of ikigai's ideas fits as a proposal and alternative to knowing the actions needed to increase the possibilities for greater physical, emotional, mental and spiritual well-being.

For the incorporation of ikigai's ideas into each other, perhaps major changes in lifestyle are not necessary. The need would be to understand the essence of the concept and incorporate it into everyday life. It would be to discover "passion", to discover that which brings happiness.

\section{INTRODUÇÃO}

Na cultura japonesa a palavra ikigai (生き甲斐), que significa “razão de viver", é um termo que faz parte do cotidiano dos japoneses e representa a motivação para viver o dia a dia.

O ideário do ikigai propagou-se mundialmente sendo muito discutido atualmente após pesquisas cientificas relacionarem estes princípios com benefícios para a saúde. Relatos da longevidade de uma população japonesa da ilha de Okinawa, do vilarejo de Ogimi seriam explicados pelo ikigai. Outro estudo levanta a possibilidade do ikigai conduzir ao encontro da prosperidade.

$\mathrm{Na}$ área da administração o conceito do ikigai está sendo utilizado para direcionamento de carreiras e aplicações motivacionais.

O presente texto pretende discutir os princípios do ikigai contribuindo em programas de qualidade de vida no ambiente profissional. 


\section{O IKIGAI}

O ikigai é intrínseco à cultura japonesa e é constituído por um eixo cognitivo e comportamental em torno do qual vários hábitos de vida e sistema de valores estão agregados e organizados. O ikigai representa a visão de vida, a sensibilidade e a maneira de agir dos japoneses ${ }^{1}$.

A discussão sobre o tema ikigai teve grande impulso com a pesquisa Ohsaki ${ }^{2}$, um estudo de coorte prospectivo que foi iniciado em $2001 \mathrm{com} 43.391$ adultos japoneses. Neste estudo, pretendeu-se associar o risco de mortalidade por todas as causas e especificidades de acordo com o sentido do ikigai.

Após 7 anos de seguimento, 3.048 pessoas haviam falecido. Observou-se que o risco de mortalidade por todas as causas foi significativamente maior entre os indivíduos que não encontraram um senso de ikigai em comparação com os indivíduos que encontraram um senso de ikigai. Quanto à mortalidade por causa específica, os indivíduos que não encontraram um senso de ikigai foram significativamente associados a um risco aumentado de doença cardiovascular e mortalidade por causa externa.

Miralles e García ${ }^{3}$, escritores e pesquisadores de desenvolvimento pessoal, entrevistaram habitantes de Ogimi, na ilha de Okinawa no Japão, cuja população apresenta grande quantidade de longevos. Esta pequena cidade tem 3 mil habitantes e está no livro Guinness World Records por ter a população mais velha do mundo na época do estudo. As pessoas dessa região do Japão tiram proveito do clima subtropical, têm uma dieta rica em frutas e vegetais, moram em comunidades onde se valorizam os laços pessoais e se mantêm fisicamente ativas por toda a vida.

Estes autores relatam que há outros lugares do mundo com condições de vida semelhantes às de Ogimi, mas não com a mesma proporção de moradores centenários. Na pesquisa realizada, os residentes de Ogimi, ao falarem sobre razões de sua longevidade, ressaltaram dieta, atividade física, resiliência e ikigai como pilares da longevidade.

Em relação à dieta, chamou a atenção o consumo menor de açúcar (a população de Okinawa consome $1 / 3$ menos de açúcar em relação ao restante do Japão) , sal (okinawanos consomem $40 \%$ menos sal que os demais japoneses), calorias diárias (1785 contra 2068) e o costume alimentar traduzido pela frase "hara hachi bu", "barriga a $80 \%$ ", ou seja, não comer até ficar empanturrado. A atividade física era comum, caracterizando-se por simples caminhadas e atividades corriqueiras como cuidar da horta ou jardim. O ponto em comum encontrado nos entrevistados foi a prática do rádio taissô (tipo de aquecimento matinal praticado em que as instruções são transmitidas via rádio). Resiliência pode ser traduzida pela persistência de uma paixão, um dos pilares do ikigai. Mesmo com algum revés, a paixão não se desfaz.

Miralles e Garcia resumem em 10 tópicos as causas da longevidade em Ogimi:

a) Manutenção da atividade (nunca se aposentar) - Em Ogimi, existe 0 consenso de que quem abandona aquilo que ama e sabe fazer, perde o 
sentido da vida. O fato de não se aposentar de suas vidas orientadas por propósitos parece ser o principal fator de longevidade e felicidade em Ogimi.

b) Ter calma - Para a população de Ogimi a pressa é inversamente proporcional à qualidade de vida.

c) Não comer até o limite - A longa digestão causa desgaste fisiológico, assim vale muito o ditado japonês "hara rati bu", que significa satisfazer a saciedade em $80 \%$.

d) Rodear-se de bons amigos - Para os cidadãos de Ogimi são os amigos que ajudam a dissolver as preocupações, proporcionam apoio e prazer pelas conversas, enfim, são responsáveis pelo sentimento de compartilhamento.

e) Colocar-se em forma para o próximo aniversário - Há a necessidade de cuidar-se. A atividade física é o caminho.

f) Sorrir - Sinônimo de relaxar.

g) Reconectar-se com a natureza - Para os cidadãos de Ogimi o contato com a natureza recarrega a bateria da alma.

h) Gratidão - Pela família, amigos, trabalho. Em Ogimi é importante visualizar o aspecto positivo da vida.

i) Viver o momento - Não se lamentar pelo passado e não temer o futuro. Fazer o melhor possível hoje para que mereça ser bem lembrado.

j) Seguir o ikigai - Para os habitantes de Ogimi todos tem uma paixão e/ou um talento único próprio que proporciona sentido à vida.

Outro pesquisador do tema ikigai, o psiquiatra Ken Mogi ${ }^{1}$, defende que viver com propósito encorajará comportamentos que desaguam na longevidade. "Ter ikigai induz você a ter um estilo de vida mais saudável, com mais exercícios, mais atividades sociais e aprendizado por toda a vida", afirma. Para Mogi é possível encontrar o ikigai nas pequenas coisas. No seu livro este autor afirma: "O mais importante é que você não pode e não deve culpar o ambiente pela falta de ikigai. Afinal, cabe a você encontrar seu próprio ikigai, do seu próprio jeito".

Segundo Mogi, após encontrar o ikigai, não há nenhuma razão para se aposentar dele. Os japoneses aparentemente não têm sequer uma palavra para "aposentadoria".

Ken Mogi aponta o que pode ajudar no encontro do ikigai:

a) Orgulho do que faz - O ikigai inicia-se ao se orgulhar daquilo que faz, produzindo sempre o melhor. A busca pela melhoria contínua é muito forte e valorizada no Japão, e é denominado de kodawari. 
b) "Estado de fluxo" - Para Ken Mogi, "estado de fluxo" é quando as pessoas estão tão envolvidas em uma atividade que nada parece importar mais. Nesta situação, a preocupação é o trabalho e não a remuneração. Ao atingir o "estado de fluxo" a preocupação é a satisfação pelos objetivos.

c) Harmonia e sustentabilidade - Mogi justifica que a sustentabilidade sem a harmonia não é viável. A busca da sustentabilidade deve ocorrer em harmonia com a família, amigos, comunidade e com o meio ambiente.

d) A alegria das pequenas coisas - O ikigai pode vir de ações como ingerir o alimento favorito logo depois de acordar ou de conversar amenidades com amigos valorizando e cultuando a "a alegria das pequenas coisas".

A discussão dos princípios do ikigai passa pela logoterapia ${ }^{4}$, conhecida também como a "psicoterapia do sentido da vida" ou, ainda, a "terceira escola vienense de psicoterapia".

Esta teoria de Viktor Emil Frankl (1905-1997) concebe uma visão do homem propondo a compreensão da existência mediante fenômenos especificamente humanos.

Este psiquiatra pesquisador reconhece a preocupação do homem com um equilíbrio interno, numa perene busca pela cessação de tensão, como objetivo maior da gratificação dos instintos e da satisfação das necessidades, constituindo-se, assim, o fim de toda atividade que envolva a vida.

Para Frankl, o que de fato impulsiona o homem não é nem a vontade de poder, nem a vontade de prazer, mas sim a vontade de sentido. Para Frankl, o homem só se torna homem e só é completamente ele mesmo quando fica absorvido pela dedicação a uma tarefa, quando se esquece de si mesmo a serviço de uma causa, ou no amor a uma pessoa.

Na logoterapia, o sentido tem um caráter objetivo de exigência e está no mundo, não no homem que a vivência.

Frankl conceitua que a vontade de sentido é orientada para uma realização de sentido, a qual provê uma razão para a felicidade, isto é, "com uma razão para ser feliz, a felicidade surge automaticamente como efeito colateral".

Ao falar de sentido, estamos fazendo referência ao significado, à coerência, à busca de propósito e finalidade. Frankl expressa que o homem que perdeu o sentido cai num vazio existencial e sofre; esta frustração existencial pode desembocar em uma sintomatologia neurótica e doentia. O sentido não é moldado pela mente, mas a mente pelo sentido. Em vez de criar um sentido, a mente tem de submeter-se a ele, uma vez encontrado.

Segundo Frankl, quatro fatores podem levar a pessoa a encontrar um sentido para a vida: 
a) as experiências - a valorização do que é importante para a pessoa, ou seja, aquilo que teve significado durante a vida, desde os pequenos até os grandes eventos;

b) as escolhas - o indivíduo é responsável por cada escolha que faz ao longo da vida, inclusive diante de situações adversas (Frankl aborda o sofrimento como uma grande oportunidade de crescimento pessoal, que, no entanto, depende de como a pessoa o enfrenta: ela pode sucumbir à dor, ou extrair ensinamentos da situação difícil);

c) responsabilidade - por tudo o que a pessoa faz, pelas escolhas e decisões;

d) significado imediato - dar sentido às coisas que acontecem na vida diária, tanto as experiências positivas, quanto as negativas.

Segundo Frankl, o sentido da vida simplesmente existe: trata-se apenas de encontrá-lo. O homem procura não é a felicidade em si, mas uma razão para ser feliz.

\section{OS PROGRAMAS DE QUALIDADE DE VIDA NO TRABALHO}

Verifica-se atualmente que um número crescente de organizações vem desenvolvendo programas para a qualidade de vida para seus trabalhadores ${ }^{5-}$ 15 .

As organizações compreenderam que, além de auxiliarem no cumprimento de suas responsabilidades sociais, tais programas são excelentes ferramentas gerenciais.

Organizações que assumem essas responsabilidades fazem um duplo investimento. O primeiro, sobre os empregados que se sentem valorizados e mantém um bom desempenho. O segundo, sobre a saúde dos trabalhadores possibilitando a redução do absenteísmo.

Os programas de qualidade de vida no trabalho estão vinculados aos programas de segurança e saúde ocupacional desenvolvidos nas organizações. Através dos resultados observados nestes programas, sobretudo no Programa de Controle Médico de Saúde Ocupacional (PCMSO) ${ }^{16}$, são propostos os programas de qualidade de vida no ambiente profissional. Como exemplos podem ser citados:

a) Programa de controle do peso - A constatação de expressivo número de trabalhadores com sobrepeso merecerá o desenvolvimento de programa desenvolvido pelo serviço de nutrição e dietética para oferecimento de dietas hipocalóricas aos trabalhadores. A instituição poderá oferecer também programas de atividade física para auxiliar no controle de peso. 
b) Programa de promoção à saúde mental - $O$ enfoque dos aspectos emocionais, por meio da disponibilização de apoio psicológico aos trabalhadores de serviços de saúde, pode ser importante ferramenta para o controle dos agentes de natureza psicossocial e organizacional permitindo a prevenção dos transtornos mentais.

c) Programa de promoção de prática de hábitos saudáveis (atividade física regular, alimentação saudável e lazer) - O incentivo ao autocuidado pela prática de atividade física regular, alimentação saudável, controle do stress e controle médico regular pode retardar declínios funcionais, além de diminuir o aparecimento de doenças crônicas. Enfatizar que uma atividade física regular e moderada reduz o risco de morte por problemas cardíacos em 20 a 25\% em pessoas com cardiopatia diagnosticada. Também pode reduzir substancialmente a gravidade de deficiências associadas às doenças cardíacas e outras doenças crônicas ${ }^{17}$. A conscientização da alimentação saudável ${ }^{17}$ enfocando o consumo correto de calorias é muito importante para a profilaxia de doenças crônicas. Dietas com excesso de gordura saturada e sal, pobres em frutas, legumes, verduras, fibras e vitaminas são fatores predisponentes para patologias como diabetes, doenças cardiovasculares, hipertensão arterial, artrites e alguns tipos de câncer. O lazer ${ }^{17}$, através de programas privilegiando atividades artísticas e culturais, pode atuar como ferramentas de profilaxia aos riscos associados a agente psicossocial e organizacional, e fortalecimento do espírito de equipe dos trabalhadores de uma instituição. Atividades como concursos para revelação de talentos existentes na empresa tem demonstrado importantes resultados na melhoria do clima organizacional.

d) Programa de apoio para abandono do hábito de fumar - Ação como o incentivo ao abandono do cigarro ${ }^{17}$ pode ser importante instrumento para a qualidade de vida do trabalhador considerando-se que 0 tabagismo é fator de risco de doença não transmissível modificável e representa a causa de morte prematura mais evitável. Importante conscientizar o trabalhador de que deixar de fumar representa a diminuição do risco de câncer de pulmão, da perda funcional, prevenindo ainda malefícios como diminuição da densidade óssea, da força muscular e da função respiratória. A empresa necessitará assumir que os custos decorrentes para a efetivação do abandono do fumo se trata de um investimento, cujo retorno ocorrerá pela melhoria da saúde do trabalhador.

e) Programa de apoio para abandono do uso de álcool - Programas para alcoolistas ${ }^{17}$ podem representar importante auxílio para trabalhadores que individualmente não conseguem controle da ingestão etílica. Registre-se que o alcoolismo impacta agudamente no absenteísmo, devido aos efeitos proporcionados pelo consumo. Este vício impacta também ao longo do tempo envolvendo doenças relacionadas ao álcool, como desnutrição e doenças do pâncreas, estômago e fígado. 
f) Programa de educação financeira - Programas de educação financeira ${ }^{17}$ podem ter valia para os trabalhadores de serviços de saúde no subsídio de planejamento de patrimônio e reserva econômico-financeira futura e para a aposentadoria.

g) Programa de apoio à mãe trabalhadora - Projetos como creches junto ao ambiente de trabalho podem constituir diferencial na fidelização de servidores, além de proporcionar tranquilidade aos pais que podem contar com filhos próximos ao ambiente de trabalho.

h) Programa de preparação para a aposentadoria - Os chamados PPA ${ }^{18}$, realizando reflexões sobre as expectativas e ansiedades pelas quais passa o trabalhador no período anterior à aposentadoria, possibilitam importante benéfico aos trabalhadores na orientação sobre alternativas, condições e meios para uma aposentadoria prazerosa e saudável.

O trabalhador nos programas de preparação para a aposentadoria deve ser conscientizado de que, apesar da cessação do ciclo produtivo profissional, o aposentado pode ser útil em outras funções à sociedade.

Nos PPA, tem lugar comum a expectativa sobre saúde física e psicológica própria do envelhecimento e os aspectos jurídico-legais relativos à aposentadoria. Assim, palestras com médicos geriatras, psicólogos, assistentes sociais, como também especialistas em legislação relativa à aposentadoria $e$ advogados especializados na área previdenciária pública e privada são importantes ao programa.

O Quadro 1 representa possível modelo para aplicação do PPA ${ }^{19}$. O diagrama contém vários módulos cabendo à empresa optar por fazer customização necessária. 


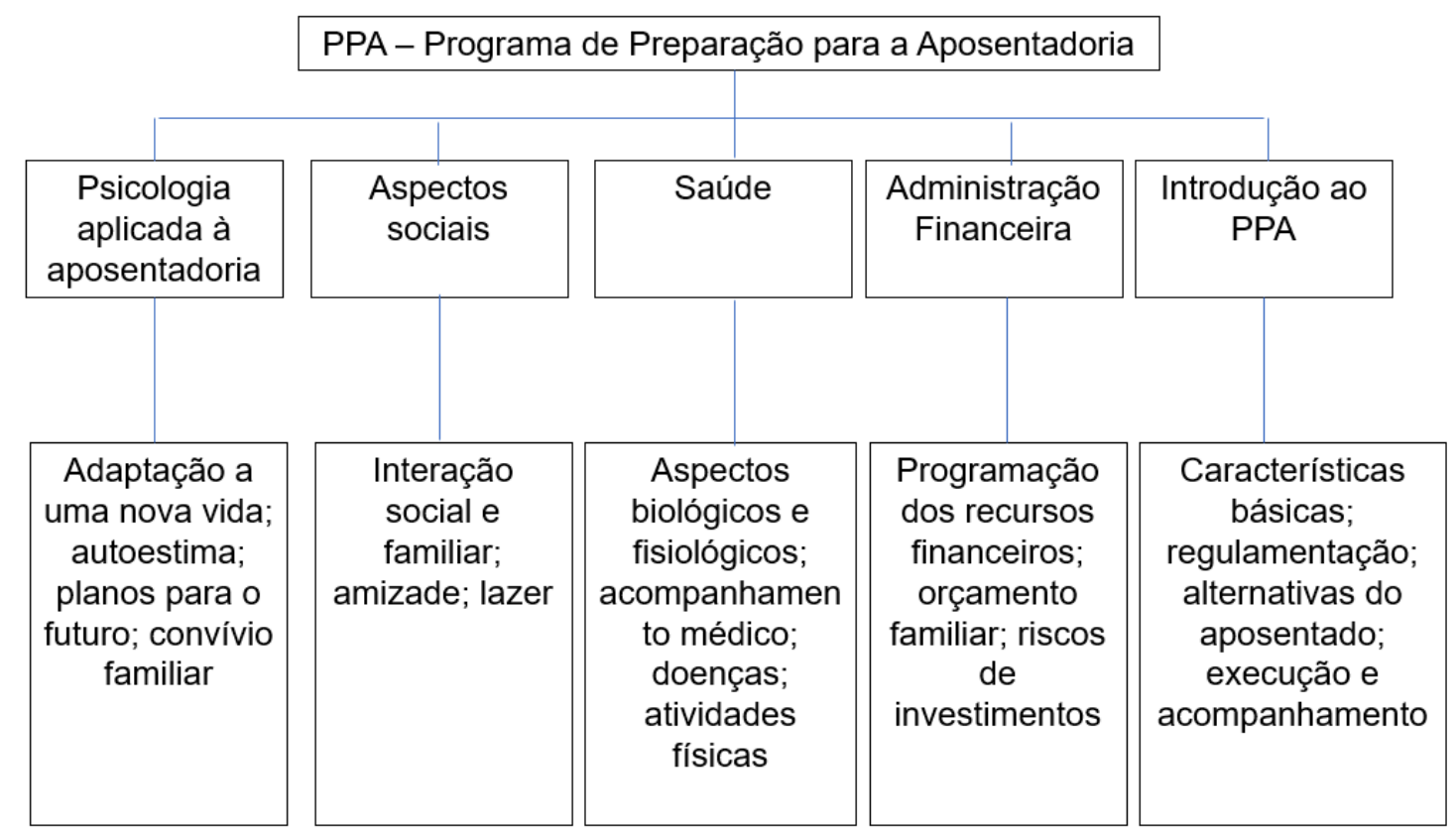

Quadro 1. Programas de preparação para aposentadoria. Fonte: Administração de Recursos Humanos. Carvalho AV, Serafim OCG

Propõe-se ainda que o desenvolvimento de um PPA seja um processo contínuo, podendo ser discutido não só pelos próximos da aposentadoria como também pelos iniciantes no mercado de trabalho.

A existência e o desenvolvimento de um PPA refletem na imagem da empresa perante o funcionário e a sociedade. O programa possibilita a revisão de conceitos sobre aposentadoria e permite foco em questões de saúde, hábitos, rotinas e estilos de vida possibilitando a construção ou o aprimoramento de um projeto de vida pessoal ou profissional.

\section{DISCUSSÃO}

Os princípios do ikigai podem contribuir para os programas de qualidade de vida no trabalho.

Marc Winn propôs em 2014 um diagrama para representar o ikigai (Figura 1) 20 . Esta figura é composta por quatro círculos onde na intersecção, ao centro, encontra-se o ikigai. Os quatro círculos representam os quatro princípios: a paixão, a missão, a profissão e a vocação. A paixão pode ser percebida ao se responder à pergunta: o que você ama? A missão vem como resposta à pergunta: do que o mundo precisa? A profissão e os talentos pessoais e profissionais são revelados respondendo: em que você é bom? Conhece-se a vocação respondendo à: por qual tarefa você gostaria de ser pago para fazer? 
Assim, o ikigai seria uma vida plena envolvendo a grande parte do tempo e energia com algo que se ama, que se faz bem, do que o mundo precisa e ainda se obtém a sustentabilidade econômico-financeira.

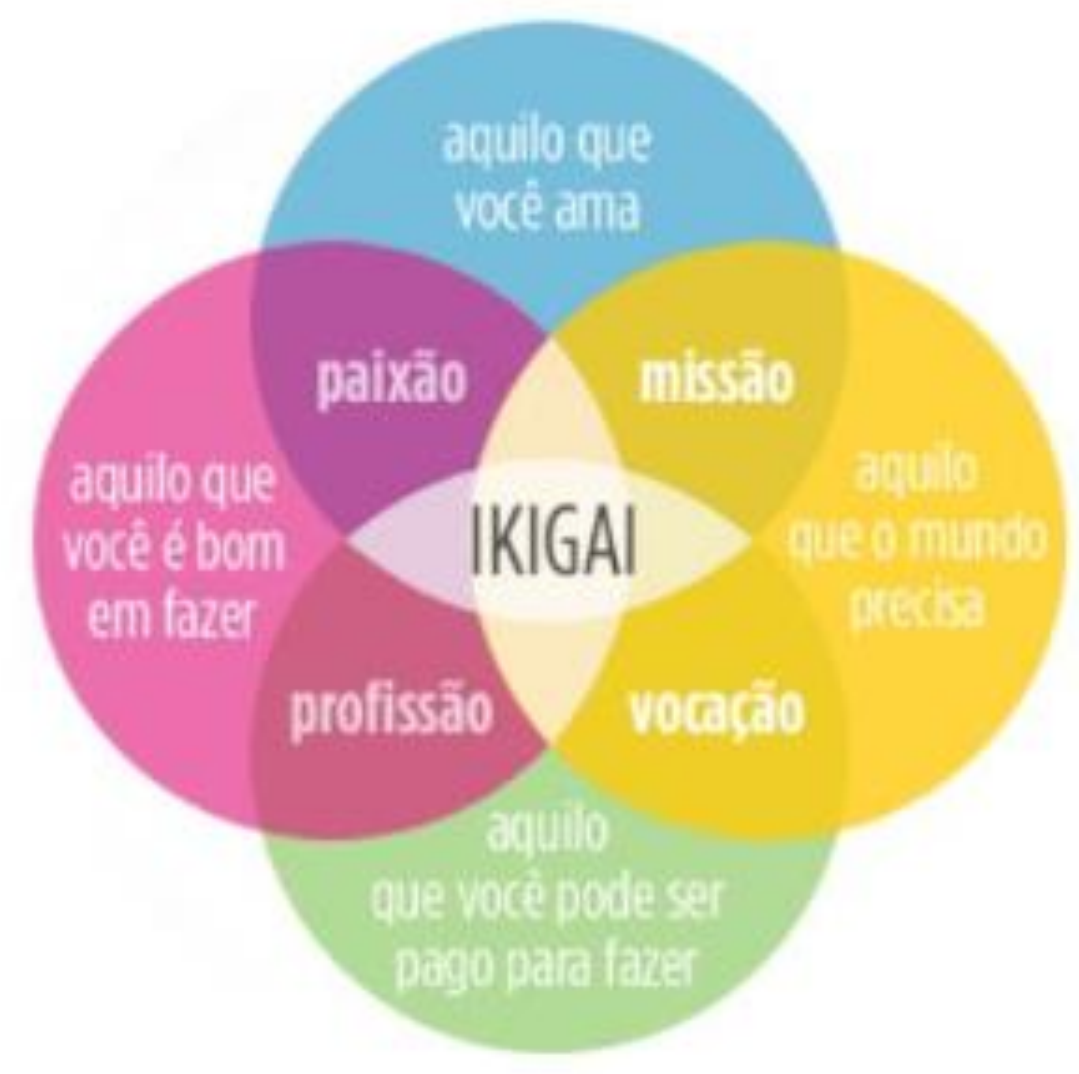

Figura 1. Diagrama representativo do ikigai, segundo Marc Winn.

Seria então possível a representação do Ikigai através de um modelo matemático?

O ideário do ikigai pode transcender um diagrama, já que não se trata de definir o ser humano por meio apenas de seu trabalho, do que ama, da vocação ou de uma suposta missão. $\mathrm{O}$ ikigai necessita conter a visão mais humana, na qual se encontra sentido e satisfação nas realizações e que pode ter implicações da cultura onde se vive. Repetindo Mogi, o ikigai é intrínseco à cultura japonesa.

Porém, é aceitável relacionar longevidade ao ikigai. As observações obtidas por Miralles e Garcia em Ogimi, vilarejo que apresenta mais longevos num país com alta taxa de expectativa de vida, pode indicar uma relação entre ikigai e estilo de vida próprio de Ogimi.

Possível concordar com Mogi quando este associa o ikigai para se encontrar a prosperidade e consequente felicidade. Para este autor, o ikigai seria um estilo 
de vida com harmonia e satisfação nas diferentes áreas da vida possibilitando alcançar a razão/propósito de vida/êxito para o dia a dia.

Necessário ainda referendar os pressupostos de Frankl, quando refere que a busca de sentido na vida (vontade de sentido) do homem é a principal força motivadora no ser humano. Para a logoterapia, a busca do homem por um sentido é a motivação primária em sua vida. Esse sentido é exclusivo e específico, uma vez que precisa e pode ser cumprido somente por esta pessoa, difere de pessoa para pessoa, de um dia para outro, de uma hora para outra.

Então, quando a Organização Mundial da Saúde (OMS) conceitua a qualidade de vida como "a percepção do indivíduo de sua posição na vida, no contexto da cultura e sistema de valores nos quais ele vive e em relação aos seus objetivos, expectativas, padrões e preocupações" e trata como conceito amplo no qual aspectos variados da vida estão envolvidos de forma complexa, tais como saúde física, estado psicológico, nível de independência, crenças pessoais e suas relações com o meio em que vive. A qualidade de vida no trabalho, por extensão, envolve a manutenção e preservação da saúde física, incluindo a garantia de condições saudáveis e seguras de (e no) trabalho.

Assim, no desenvolvimento de programas de qualidade de vida no trabalho, em especial no PPA, onde os trabalhadores têm a possibilidade de refletir sobre 0 momento presente sem esquecer a futura cessação do período produtivo, preparando-se com informações para o envelhecimento, a discussão do ideário do ikigai cabe como proposta e alternativa para conhecimento das ações necessárias para aumentar as possibilidades de maior bem estar físico, emocional, mental e espiritual.

\section{CONCLUSÃO}

Ikigai pode ser entendido como um ideal de vida onde resultados dependem de variáveis como clima, hábitos alimentares, atividade física, relações e apoios sociais e familiares propiciando qualidade de vida e longevidade. Valores pessoais e da comunidade envolvida contam e tornam-se componentes importantes no ideário do ikigai de cada pessoa.

Desejável seria se todos pudessem contar com o clima, a alimentação disponível e o convívio social existente em Ogimi possibilitando assim a probabilidade de uma longevidade com qualidade de vida.

A pergunta seria então: podemos criar nossa Ogimi? Para a incorporação do ideário do ikigai em cada um talvez não sejam necessárias grandes mudanças no estilo de vida. A necessidade seria entender a essência do conceito e incorporá-lo no cotidiano. Seria descobrir a "paixão", descobrir aquilo que traz felicidade. 


\section{REFERÊNCIAS}

1. Mogi K. IKIGAI: Os cinco passos para encontrar seus propósitos de vida e ser mais feliz. 1ํㅡㄹ ed. Bauru SP: Astral Cultural; 2018.

2. Sone T, Nakaya N, Ohmori K, Shimazu T, Higashiguchi M, Kakizaki M, Kikuchi N, Kuriyama S, Tsuji I. Sense of Life Worth Living (Ikigai) and Mortality in Japan: Ohsaki Study. Psychosom Med. 2008 Jul;70(6):70915. DOI: $10.1097 /$ PSY.0b013e31817e7e64

3. Garcia H. Ikigai: Os segredos dos japoneses para uma vida longa e feliz. $1^{\underline{a}}$ ed. Rio de Janeiro: Intrínseca; 2018.

4. Pereira IS. A vontade de sentido na obra de Viktor Frankl. Psicologia USP, 18(1), 125-136 DOI: 10.1590/S0103-65642007000100007

5. WHO. The World Health Organization Quality of Life assessment (WHOQOL): position paper from the World Health Organization. Soc Sci Med. 1995 Nov;41(10):1403-9. Disponível em https://www.ncbi.nlm.nih.gov/pubmed/8560308 (acesso em 14 jan. 2019)

6. Rodrigues MVC. Qualidade de vida no trabalho: evolução e análise no nível gerencial. UNIFOR, Fortaleza, 1991.

7. Walton RE. Quality of Working Life: What is it? Sloan Management, 15(1): 11- 21,1973.

8. Fernandes EC. Qualidade de vida no trabalho: como medir para melhorar. Salvador: Casa da Qualidade, 1996.

9. Dantas J. Coração e fatores de risco. Qualidade total na promoção da Saúde. 1a. ed. Belo Horizonte: Editora o Lutador, 1996. v. 1. 168p.

10. França ACL. Indicadores empresariais de qualidade de vida no trabalho esforço empresarial e satisfação dos empregados no ambiente de manufaturas com certificação ISO 9000. Tese de Doutorado em Administração - USP,1996.

11. França ACL. Qualidade de vida no trabalho: conceitos, abordagens, inovações e desafios nas empresas brasileiras. Vol. 1. Rio de Janeiro: [s.n.], 1997.

12. Albuquerque LG, Limongi-França AC. Estratégia de Recursos Humanos e Gestão de Qualidade de Vida no Trabalho: O stress e a expansão do conceito de qualidade total. Curso Avançado de Gestão Empresarial em Qualidade de Vida: FEA/USP, São Paulo, 2003. Disponível também na Revista de Administração São Paulo, v. 33, no 2, p.40-51, abr - jun de 1998. 
13. Limongi-França AC, Zaima G. Gestão de Qualidade de Vida no Trabalho - GQVT. In: Manual de Gestão de pessoas e equipes: estratégias e tendências. São Paulo: Gente, 2002.

14. Chiavenato I, Matos FG. Visão e Ação Estratégica. São Paulo: Prentice Hall, 2002.

15. Herzberg F. "One More Time: How Do You Motivate Employees?". Harvard Business Review. 65, September - October 1987.

16. Normas Regulamentadoras. Disponível em http://www.tst.jus.br/web/trabalhoseguro/normas. (acesso em 14 jan. 2019).

17. WHO. WORLD HEALTH ORGANIZATION. Envelhecimento ativo: uma política de saúde / World Health Organization; tradução Suzana Gontijo. - Brasília: Organização Pan-Americana da Saúde, 2005. 60p. Disponível em: http://bvsms.saude.gov.br/bvs/publicacoes/envelhecimento ativo.pdf (acesso em 14 jan. 2019)

18. Murta SG, Abreu S, França CL, Pedralho M, Seidl J, Lira NPM, Carvalhedo RKM, Conceição AC, Gunther IA. Preparação para a aposentadoria: implantação e avaliação do programa viva mais!. Psicologia: Reflexão e Crítica. 2014; 27(1): 01-09. DOI: https://dx.doi.org/10.1590/S0102-79722014000100001

19. Carvalho AV. Serafim OCG. Administração de Recursos Humanos. Volume 2 CHIAVENATO, Idalberto Recursos Humanos. 3ª Edição LUCENA, Maria Diva da Salete. Planejamento de Recursos Humanos. Ed. Altas. MORVAN, de Mello Moreira. Determinantes Demográficos do Envelhecimento Brasileiro Preparação para a aposentadoria: Desafios a enfrentar Lucia França 2002. Disponível em:

http://www.luciafranca.com/PDF/Aposentadoria\%20Article\%20Portugues .pdf. (acessado em 26/05/2019)

20. Rmcholewa. A Origem do Famoso Gráfico de Venn do Ikigai. https://rmcholewa.com/2018/12/20/o-famoso-grafico-de-venn-do-ikigai/ (acessado em 18 jul. 2019)

Recebido: 02 de setembro de 2019. Aceito: 09 de setembro de 2019

Correspondência: Milton Osaki. Av. Dr. Arnaldo, 351, 2o andar - Pacaembu São Paulo SP - CEP: 01246-000. E-mail: osaki.milton@gmail.com 
Conflito de Interesses: os autores declararam não haver conflito de interesses.

(C) This is an Open Access article distributed under the terms of the Creative Commons Attribution License, which permits unrestricted use, distribution, and reproduction in any medium, provided the original work is properly cited 